\title{
El perfil del estudiante universitario croata de estudios hispánicos
}

\author{
Andrea-Beata Jelić \\ Facultad de Humanidades y Ciencias Sociales \\ Universidad de Zagreb \\ abjelic@ffzg.hr
}

\begin{abstract}
En los últimos años en el campo de la adquisición de lenguas extranjeras se han realizado varios estudios que describen el perfil de los estudiantes universitarios con el fin de adaptar los currículos y la enseñanza a sus características y necesidades. El presente artículo tiene como objetivo identificar varios componentes del perfil del estudiante universitario inscrito en el programa de estudios hispánicos de la Facultad de Humanidades y Ciencias Sociales de la Universidad de Zagreb. Los resultados obtenidos permiten entender mejor esta población estudiantil, lo que abre nuevas líneas de investigación y puede servir de base para realizar algunos cambios en la práctica docente universitaria.
\end{abstract}

Palabras clave: perfil del estudiante universitario, factores individuales, los alumnos de ELE.

\section{Introducción}

La noción de la enseñanza centrada en el alumno ha estado presente en la didáctica de lenguas extranjeras desde hace unos cincuenta años. Desde entonces se pretenden revelar sus características individuales y sus necesidades como individuos dentro de un contexto social. La identificación del perfil de los alumnos presenta una herramienta tanto experimental como práctica para lograr este objetivo. Las investigaciones a menudo se centran en un menor número de características e identifican perfiles parciales de los aprendientes, pero hay también estudios que establecen un perfil más complejo de los alumnos abarcando varios grupos de factores (Cea Álvarez 2015; Marijuán Adrián 2011; Rubio Martín 2011). En todos los estudios se subraya la importancia de esas reflexiones y datos en el desarrollo y la implementación de un currículo adaptado a las necesidades, aptitudes y actitudes de los alumnos. 


\section{El perfil del estudiante croata de estudios hispánicos}

Teniendo en cuenta que hasta hoy se han investigado solo perfiles parciales de los alumnos croatas de español lengua extranjera (ELE), como por ejemplo sus rasgos cognitivos (Hadrović 2017; Jeleč 2018) y afectivos (Jelić/Bajt 2016; Mandić 2017), nos proponemos presentar los resultados de la investigación realizada con los estudiantes de estudios hispánicos con el fin de presentar un perfil más completo de esos alumnos.

\subsection{Objetivos de la investigación}

El presente estudio tiene como objetivo identificar el perfil del estudiante universitario croata de estudios hispánicos de la Universidad de Zagreb. Más específicamente, se desea analizar (a) el componente demográfico, (b) el éxito académico y las características (c) lingüísticas, (d) cognitivas y (e) afectivas del perfil estudiantil. Se pretende entender mejor esta población, especialmente en cuanto a las posibles diferencias entre los estudiantes de grado y de máster. A base de resultados se propondrán algunos cambios en la práctica docente universitaria en este contexto.

\subsection{Metodología}

\subsubsection{Participantes, instrumentos y procedimiento}

La muestra incluye 102 estudiantes de estudios hispánicos en la Facultad de Humanidades y Ciencias Sociales de la Universidad de Zagreb, tanto al nivel de grado $(\mathrm{N}=75)$ como al nivel de máster $(\mathrm{N}=27)$.

La investigación se llevó a cabo con un cuestionario elaborado para este estudio que cubre cinco componentes del perfil estudiantil. La parte del cuestionario que se refiere al componente demográfico es descriptiva y consta de unas quince preguntas sobre varios datos del estudiante, la parte que abarca el éxito académico incluye los datos sobre las notas obtenidas durante el programa de estudios y la parte que se refiere al componente lingüístico incluye una autoevaluación de las cuatro destrezas lingüísticas (MCER 2002). Para investigar el componente cognitivo se utilizó un cuestionario sobre las estrategias de aprendizaje de la LE (adaptado de García Herrero/Jiménez Vivas 2014) que los participantes tenían que calificar en una escala Likert desde 1 (no estoy de acuerdo) hasta 5 (estoy totalmente de acuerdo) puntos. Se midieron las estrategias de memorización (6 ítems, $\alpha=0,52$ ), las estrategias cognitivas (13 ítems, $\alpha=0,74$ ), las estrategias compensatorias ( 5 ítems, $\alpha=0,47$ ) y las estrategias metacognitivas (9 ítems, $\alpha=0,78$ ). Para investigar el componente afectivo se utilizó un cuestionario sobre la autopercepción de la motivación académica personal (Boza Careño/Toscano Cruz 2012). Se midieron las metas vitales (22 ítems, $\alpha=0,85$ ), los motivos personales (20 ítems, $\alpha=0,89)$ y la implicación en el estudio (10 ítems, $\alpha=0,69)$. El cuestionario fue realizado en croata durante las clases universitarias regulares. El análisis de los datos se hizo en el programa SPSS 24. 


\subsection{Resultados}

En cuanto al componente demográfico, los participantes son jóvenes entre 19 y 26 años, mayoritariamente de sexo femenino (93\%). La mayoría (62\%) lleva estudiando español entre 3 y 7 años, años, 23\% más de 7 años y 15\% entre 1 y 2 años. El 73\% de los estudiantes ha tenido clases de español en la escuela secundaria, $23 \%$ en escuelas de idiomas y un $4 \%$ lo ha estudiado de otra manera. Para inscribirse en el programa de estudios hispánicos la mayoría ha aprobado el examen final de educación secundaria (57\%) o el examen de conocimiento de español administrado por la Facultad de Humanidades y Ciencias Sociales (39\%), mientras que solo $2 \%$ de los estudiantes han aprobado un examen DELE que les califica a inscribirse. La inscripción en la carrera de estudios hispánicos fue la primera opción para el $86 \%$ de los estudiantes y al seleccionar estos estudios $78 \%$ de ellos reflexionaron sobre su futuro trabajo. Al nivel de máster, $80 \%$ de los estudiantes eligieron el profesorado (57\%) de ELE o el traductorado (43\%). Junto al español, 79\% de los estudiantes estudian en la facultad otra lengua extranjera. Entre los estudiantes $44 \%$ han visitado un país de habla española y para la mayoría (71\%) la estancia duró entre una semana y dos meses.

El éxito académico de los estudiantes se midió mediante tres tipos de notas que recibieron durante sus estudios. La nota media de todas las asignaturas de estudio en una escala entre 1 y 5 es $M=3,96(\mathrm{DE}=0,49)$, la de las asignaturas hispánicas es $\mathrm{M}=3,94(\mathrm{DE}=0,63)$ y la de la asignatura Ejercicios de lengua española $\mathrm{M}=3,72(\mathrm{DE}=0,79)$. Para explorar si existe una diferencia entre los estudiantes de grado y de máster, se usó la prueba t que revela una diferencia estadísticamente significativa solo en cuanto a la nota media de todas las asignaturas del estudio $(\mathrm{p}<0,05)$ en favor de los estudiantes de máster.

Con el fin de analizar el componente lingüístico, se pidió a los estudiantes que se autoevaluaran según los niveles del MCER (2002). Los resultados de la autoevaluación de las destrezas receptivas y productivas se presentan en el Gráfico 1.

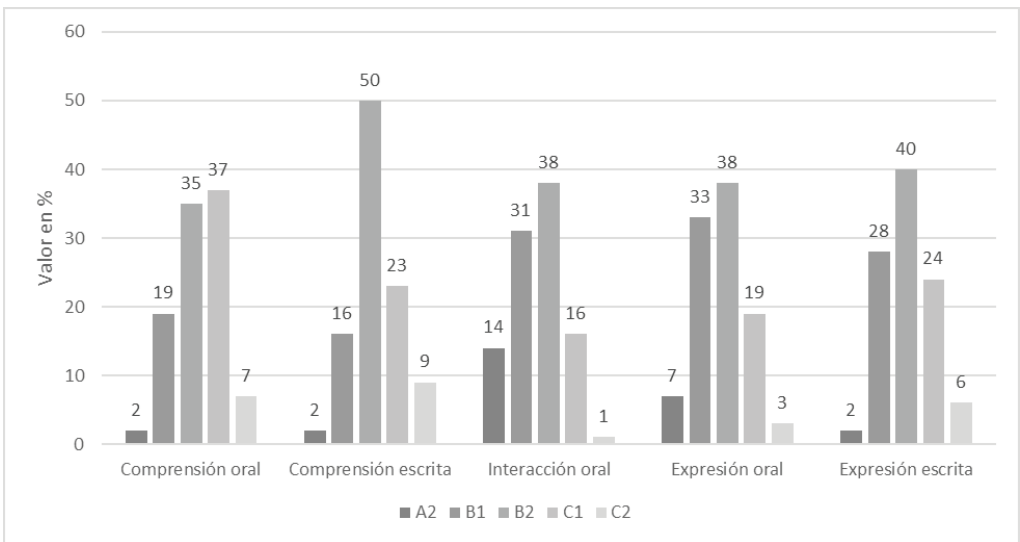

Gráfico 1. Autoevaluación de las destrezas lingüísticas según los niveles del MCER (2002) 
La mayoría de los estudiantes se atribuyen los niveles más altos de competencia (un nivel B2 o superior) en la comprensión escrita (82\%), seguida de la comprensión oral (79\%), la expresión escrita (70\%) y la producción oral $(60 \%$ en la expresión oral y 55\% en la interacción oral). La prueba de chi-cuadrado revela una diferencia estadísticamente significativa entre los estudiantes de grado y de máster en cuanto a todas las destrezas autoevaluadas: la comprensión oral $\left(\chi^{2}=25,26 ; \mathrm{df}=4 ; \mathrm{p}<0,001\right)$, la comprensión escrita $\left(\chi^{2}=48,21 ; \mathrm{df}=4 ; \mathrm{p}<0,001\right)$, la interacción oral $\left(\chi^{2}=47,45 ; \mathrm{df}=4 ; \mathrm{p}<0,001\right)$, la expresión oral $\left(\chi^{2}=39,07 ; \mathrm{df}=4\right.$; $\mathrm{p}<0,001)$ y la producción escrita $\left(\chi^{2}=34,66 ; \mathrm{df}=4 ; \mathrm{p}<0,001\right)$ en favor de los estudiantes de máster.

El componente cognitivo se analizó mediante cuatro tipos de estrategias de aprendizaje que reflejan los procesos cognitivos de los aprendientes de una LE. Las estrategias más utilizadas son las estrategias metacognitivas $(M=3,69 ; \mathrm{DE}=0,62)$, seguidas por las estrategias compensatorias $(\mathrm{M}=3,36 ; \mathrm{DE}=0,62)$ y cognitivas $(\mathrm{M}=3,22 ; \mathrm{DE}=0,62)$. Las estrategias menos utilizadas son las de memorización $(\mathrm{M}=2,70 ; \mathrm{DE}=0,64)$. En cuanto a la diferencia en el uso de las estrategias de aprendizaje entre los estudiantes de grado y de máster, la prueba t revela que hay una diferencia estadísticamente significativa respecto a las estrategias cognitivas $(\mathrm{t}=-2,715, \mathrm{df}=100, \mathrm{p}<0,01)$ y a las estrategias compensatorias $(\mathrm{t}=-2,386, \mathrm{df}=100$, $\mathrm{p}<0,05)$ en favor de los estudiantes de máster.

Para medir el componente afectivo se analizó la autopercepción de la motivación académica personal de los participantes mediante sus metas vitales, motivos personales y la implicación en el estudio. Los estudiantes están bastante implicados en sus estudios $(M=3,89 ; \mathrm{DE}=0,54)$ y tienen un nivel medio hacia alto de motivación personal para el estudio $(\mathrm{M}=3,88 ; \mathrm{DE}=0,67)$. También tienen bastante desarrollados diferentes tipos de metas vitales $(M=3,44 ; D E=0,53)$. La prueba t no revela una diferencia estadísticamente significativa entre los resultados de los estudiantes de grado y los estudiantes de máster.

\subsection{Discusión}

Nuestros estudiantes son jóvenes adultos, mayoritariamente de sexo femenino que llevan estudiando español en promedio siete años. Comienzan su aprendizaje a menudo antes de inscribirse en la universidad y la escuela secundaria se revela como el entorno más importante que despierta su interés por la lengua y les motiva a elegir los estudios hispánicos. Para la mayoría de los estudiantes los estudios hispánicos son la primera opción en la selección de carrera. También, tienen cierta visión de su futuro trabajo, lo que puede explicar su mayor interés por el máster en didáctica de ELE o en traducción, que llevan a profesiones reguladas, que por el máster en literatura o lingüística hispánica. Durante los estudios los estudiantes son bastante exitosos tanto en las asignaturas hispánicas como en sus otras asignaturas. Es la asignatura Ejercicios de lengua española la que les resulta hasta cierto punto más difícil que las demás asignaturas. Podemos suponer que esto se debe a que en esta asignatura no solo se trabaja, sino también se evalúa directa 
y exclusivamente su competencia comunicativa, lo que para ellos representa el mayor desafío ya que se trata de la competencia central que desarrollan durante sus estudios. La mayoría de los encuestados se encuentran en el nivel B1/B2 del español y estiman que tienen más desarrolladas las destrezas receptivas que las destrezas productivas, mientras que es en la destreza de interacción oral en la que se sienten menos competentes. Muestran también una competencia estratégica bastante desarrollada, siendo las estrategias metacognitivas y las estrategias compensatorias las utilizadas más frecuentemente. El uso de estrategias metacognitivas normalmente caracteriza a los estudiantes exitosos puesto que la metacognición se considera fundamental para un aprendizaje autorregulado y consciente. El uso de las estrategias compensatorias muestra que los estudiantes han aprendido a eliminar los vacíos en diferentes componentes de su competencia y se esfuerzan en conseguir sus objetivos comunicativos. Los aspectos afectivos juegan un papel importante para todos los estudiantes de nuestro estudio. Por un lado, los estudiantes muestran un grado medio hasta alto de motivación, lo que les incita a implicarse en el estudio y crear condiciones favorables para el aprendizaje. Por otro, tienen bastante desarrolladas sus metas vitales al igual que una actitud positiva hacia el estudio y, en última instancia, la obtención de un diploma universitario, objetivo que estiman importante para su vida en general.

Los estudiantes muestran también un desarrollo significativo en casi todos los componentes analizados del perfil. Primero, los estudiantes de máster superan a sus colegas de grado en cuanto a su éxito académico general. Además, los participantes demuestran una progresión lingüística en todas las destrezas lingüísticas. Finalmente, el componente cognitivo del aprendizaje universitario cambia también sobre todo en el uso de estrategias cognitivas y compensatorias, donde los estudiantes de máster sobresalen a sus colegas de grado. El manejo cognitivo de los contenidos a aprender es ciertamente un elemento que les permitirá ser más eficaces en su formación continua como jóvenes profesionales en sus futuras carreras, mientras que las estrategias compensatorias les ayudarán a seguir desarrollando su competencia comunicativa al salir de la universidad.

En cuanto a la práctica docente universitaria, parece que el desarrollo ulterior de la competencia comunicativa de los estudiantes queda como uno de los objetivos principales de la enseñanza en este entorno. Las destrezas productivas, y especialmente la destreza de producción oral, deberían estar aún más presentes en los contenidos del currículo y se deberían organizar formas de trabajo más diversificadas que proporcionen el uso natural de la lengua oral y escrita. Los intercambios informales y la interacción con los locutores nativos (los profesores, pero también, por ejemplo, los estudiantes extranjeros de habla hispana que vienen a Zagreb dentro de los programas de colaboración internacional) podrían desarrollarse dentro de clubs de estudiantes o grupos de interés bajo los auspicios de las estructuras formales universitarias. También, una oferta de becas patrocinada con la ayuda de varios organismos fuera de la universidad para atender cursos lingüísticos, sobre todo en España, sería también beneficiosa. El rol primordial de cualquier programa de estudios universitarios es preparar a los estudiantes para su futuro trabajo y el mercado laboral. En este sentido, 
parece necesaria una reflexión más detallada sobre los contenidos ofrecidos al nivel de máster de estudios hispánicos.

\section{Conclusión}

La identificación del perfil de los estudiantes de una LE proporciona múltiples datos sobre las características individuales de los alumnos, que juegan un rol importante en el proceso de aprendizaje y adquisición de esa lengua. En nuestro contexto universitario de estudios hispánicos, se revela la interacción de varios componentes del perfil estudiantil y se manifiesta un progreso en todos los elementos analizados. Un perfil afectivo favorable se muestra particularmente importante para incitar, orientar y mantener la motivación para los estudios. Visto que ese interés se despierta ya durante la educación secundaria de los estudiantes, una colaboración entre los profesores de estos dos niveles educativos sería indispensable para atraer a los estudiantes hacia los estudios hispánicos en la facultad. Los contenidos que se les ofrecen en el contexto universitario deberían también hasta cierto punto adaptarse a sus necesidades y preferencias reveladas.

Las líneas futuras de investigación pueden incluir un análisis más detallado de varios rasgos de los conocimientos lingüísticos de español de los estudiantes como prerrequisitos para cursar la carrera en filología hispana. Un entendimiento mejor de las demás características afectivas y su influencia en otros componentes del perfil estudiantil permitirá una adaptación aún mejor de la práctica docente en este entorno. Para finalizar, un análisis de las necesidades tanto de los estudiantes (en relación con su futuro trabajo) como del mercado laboral, se revela primordial para ofrecer una formación adaptada a las diversas salidas profesionales disponibles.

\section{Bibliografía}

Boza Carreño, Ángel / Toscano Cruz, María de la O (2012). Motivos, actitudes y estrategias de aprendizaje: aprendizaje motivado por alumnos universitarios, en: Profesorado, 16(1), pp. 125-142, < http://hdl.handle.net/10481/22994> (10/4/2019).

Cea Álvarez, Ana María (2015). La experiencia previa de los alumnos lusófonos de ELE: una variable a tener en cuenta, en: La enseñanza de ELE centrada en el alumno. Actas del XXV Congreso Internacional de la ASELE [coord. Yuko Morimoto / Maria Victoria Pavón / Rocio Santamaría Martínez], Málaga: Universidad de Málaga, pp. 235-243, <http://cvc.cervantes.es/ensenanza/ biblioteca_ele/asele/pdf/25/25_0235.pdf > (16/10/2019).

Consejo de Europa (2002). Marco común europeo de referencia para las lenguas (MCER), Instituto Cervantes, Anaya: Madrid.

García Herrero, Ma de las Mercedes / Jiménez Vivas, Amparo (2014). Estrategias en el aprendizaje de la lengua extranjera y niveles de competencia en estu- 
diantes universitarios de magisterio, en: Revista de Investigación Educativa, 32(2), pp. 363-378, <http://dx.doi.org/10.6018/rie.32.2.167421> (20/10/2019).

Hadrović, Monika (2017). El uso de las estrategias de aprendizaje de los estudiantes croatas de ELE en relación con el nivel de aprendizaje. Trabajo de fin de máster, Zagreb: Facultad de Humanidades y Ciencias Sociales.

Jeleč, Ivana (2018). Estilos y estrategias de aprendizaje de español como lengua extranjera. Trabajo de fin de máster, Zagreb: Facultad de Humanidades y Ciencias Sociales.

Jelić, Andrea-Beata / Bajt, Anja (2016). Orientaciones motivacionales de los estudiantes universitarios croatas de ELE, en: SRAZ, LXI, pp. 161-174.

Marijuán Adrián, Isabel (2011). Perfil del estudiante australiano de español y el profesor de ELE, en: Competencias y estrategias docentes en el contexto AsiaPacifico, Selección de artículos del II Congreso de Español como Lengua Extranjera en Asia-Pacifico, Manila: Instituto Cervantes de Manila y la Embajada de España en Filipinas, < https://cvc.cervantes.es/ensenanza/biblioteca_ele/publicaciones_centros/pdf/manila_2011/12_investigaciones_04.pdf> (16/1/2019). Mandić, Marina (2017). Ansiedad ante la lengua en alumnos croatas de español como lengua extranjera. Trabajo de fin de máster, Zagreb: Facultad de Humanidades y Ciencias Sociales.

Rubio Martín, Raquel (2011). Diseñando el perfil del estudiante universitario japonés de español como lengua extranjera/segunda lengua. Trabajo presentado en el congreso mundial de profesores de español, Instituto Cervantes, $<$ http://comprofes.es/sites/default/files/slides/rubio_martin_raquel.pdf $>$ $(16 / 1 / 2019)$.

\section{Profil hrvatskog studenta hispanistike}

Posljednjih godina u području koje se bavi procesom ovladavanja stranim jezikom provedeno je više istraživanja u kojima se opisuje profil učenika s ciljem prilagodbe kurikula i nastave njihovim karakteristikama i potrebama. Cilj je ovoga rada opisati različite sastavnice profila studenata hispanistike na Filozofskom fakultetu Sveučilišta u Zagrebu i analizirati njihove demografske, akademske jezične, kognitivne i afektivne značajke. Dobiveni rezultati omogućuju bolje razumijevanje ove studentske populacije što otvara nova istraživačka pitanja ali može predstavljati i temelj prilagodbe sveučilišne nastave. jezika.

Ključne riječi: profil studenata, individualni čimbenici, učenici španjolskog kao stranog 
